\title{
The Comparison of Endoscopic Biliary Drainage in Malignant Hilar Obstruction by Cholangiocarcinoma: Bilateral Metal Stents versus Multiple Plastic Stents
}

\author{
Jun Young Kim¹, Sang-geul Lee ${ }^{2}$, Danbee Kang ${ }^{3}$, Dong Kyu Lee², Joo Kyung Park², Kyu Taek Lee², Jong Kyun \\ Lee $^{2}$, and Kwang Hyuck Lee ${ }^{2}$ \\ 1'Department of Medicine, Samsung Changwon Hospital, Sungkyunkwan University School of Medicine, Changwon, ${ }^{2}$ Department \\ of Medicine, Samsung Medical Center, Sungkyunkwan University School of Medicine, and ${ }^{3}$ Department of Clinical Research and \\ Evaluation, Samsung Advanced Institute for Health Sciences \& Technology (SAIHST), Sungkyunkwan University, Seoul, Korea
}

\begin{abstract}
Article Info
Received August 8, 2020

Revised October 15, 2020

Accepted December 10, 2020

\section{Corresponding Author}

Kwang Hyuck Lee

ORCID https://orcid.org/0000-0002-5558-0415

E-mail lkhyuck@gmail.com
\end{abstract}

Jun Young Kim and Sang-geul Lee contributed equally to this work as first authors.

\begin{abstract}
Background/Aims: For the management of hilar malignant biliary obstruction (HMBO), endoscopic biliary drainage (EBD) is preferred over percutaneous transhepatic biliary drainage (PTBD) because of its convenience. However, there is no established guideline for malignant hilar obstruction that requires multiple stenting. In this study, we compared the efficacy of bilateral metal stents (BMS) versus multiple plastic stents (MPS).

Methods: In this retrospective study, we analyzed 102 patients who underwent EBD with either BMS or MPS due to HMBO caused by hilar cholangiocarcinoma between 1996 and 2018 at Samsung Medical Center. We compared the successful drainage rates, cholangitis events, overall complications, mortality, and conversion rates to PTBD between the two groups.

Results: The successful drainage rates in the BMS group and the MPS group were $71.4 \%$ (25/35) and 65.6\% (44/67), respectively, with no significant difference. The MPS group had a higher cholangitis risk (hazard ratio [HR], 2.08; 95\% confidence interval [Cl], 1.21 to 3.58 ) and higher 6-month mortality $(\mathrm{HR}, 2.91 ; 95 \% \mathrm{Cl}, 1.26$ to 6.71) than the BMS group. There were no significant differences in overall complications or the conversion rate to PTBD between the groups.

Conclusions: In patients with malignant $\mathrm{HMBO}$, the BMS group showed better outcomes in terms of the cholangitis rate and 6-month mortality than the MPS group. Therefore, if possible, bilateral metal stenting is recommended for $\mathrm{HMBO}$ caused by hilar cholangiocarcinoma. (Gut Liver 2021;15:922-929)
\end{abstract}

Key Words: Klatskin tumor; Cholestasis; Cholangiopancreatography, endoscopic retrograde; Stents

\section{INTRODUCTION}

The management of obstructive jaundice and cholangitis is important for improving the liver function with optimal survival outcomes and quality of life for patients with unresectable hilar malignant biliary obstruction $(\mathrm{HMBO}){ }^{1}$ For unresectable HMBO, two non-surgical methods are used for palliative drainage, endoscopic biliary drainage (EBD; internal drainage) and percutaneous transhepatic biliary drainage (PTBD; external drainage). Both methods are used complementarily and as alternatives. However, EBD is currently preferred because it is more convenient and less invasive. Therefore, the mortality rate is lower and the hospital stay is shorter in EBD patients. ${ }^{2}$

The types of stents used for EBD are classified into plastic stents and metal stents according to the composition materials. In previous studies, metal stents had a higher patency rate and a lower re-intervention rate than plastic stents. The technical success rate was not different between the two types of stents. ${ }^{3-5}$ However this evidence was well- 
established in only distal biliary obstructions and currently, metallic stents are recommended for the malignant distal bile duct obstructions. ${ }^{6-9}$ In $\mathrm{HMBO}$, there are no definite consensus guidelines about the optimal type of stent and drainage area (unilateral or bilateral drainage) and thus, controversy still exists. Generally, metal stents are recommended for biliary palliation in hilar cholangiocarcinoma patients with a predicted survival of $>3$ months, and plastic stents are recommended as temporary drainage for patients with cholangitis with an undetermined treatment plan. ${ }^{10}$ However, the consensus is based on the evidence comparing single metal and plastic stents. ${ }^{7,11-13}$ Multiple stents are also frequently needed because single stent placement is not enough for effective drainage in patients with complicated hilar biliary anatomy. ${ }^{2}$ Although several recent studies showed effective drainage in bilateral stents compared to single stents in patients with hilar cholangiocarcinoma, ${ }^{6,714-17}$ the evidence is not strong enough for a consensus recommendation. ${ }^{10}$ With recent progress in endoscopic devices and technological development, both multiple plastic stents (MPS) and bilateral metal stents (BMS) are frequently used for EBD in HMBO. However, there are few studies directly comparing the two groups.

In this study, we compared MPS and BMS for EBD in patients with HMBO caused by hilar cholangiocarcinoma.

\section{MATERIALS AND METHODS}

\section{Study design, patients, and main outcomes}

We retrospectively screened a total of 293 patients who underwent palliative EBD due to $\mathrm{HMBO}$ caused by pathologically proven hilar cholangiocarcinoma between 1996 and 2018 at Samsung Medical Center. Preoperative inoperability was determined by computed tomography, magnetic resonance imaging and positron emission tomography-computed tomography. Among these patients, we excluded 191 patients who underwent single stenting or primary PTBD. A total of 102 patients who underwent primary EBD with BMS ( $\mathrm{n}=35$ ) or MPS (two or more plastic stents, $n=67)$ were enrolled. In BMS group, uncovered selfexpandable metal stents were used and in MPS group, and routine practice of scheduled 3-month interval of regular procedure was performed. We estimated that stent patency was maintained when plastic stents were regularly changed without stent dysfunction such as interval cholangitis or obstruction events. The patients were assessed for basic demographics (age, sex, and body mass index), comorbidities, such as hypertension, diabetes and end-stage renal disease, laboratory data for liver function indexes (prothrombin time and serum albumin), and Bismuth types of hilar cholangiocarcinoma. We compared the two groups using successful drainage as the main outcome. We also compared the overall complication rates, cholangitis rates, mortality, and conversion rates to PTBD. This study protocol was conducted in accordance with the Declaration of Helsinki and approved by the Institutional Review Board of Samsung Medical Center (IRB number: 2018-11-133005). The Institutional Review Board waived the requirement for informed consent because we used de-identified data.

\section{Definitions}

Successful drainage was defined as either (1) a decrease in the total bilirubin level to $\leq 30 \%$ of the pretreatment value within 2 weeks or to $\leq 50 \%$ within 4 weeks or (2) no increase in the total bilirubin level over $1 \mathrm{mg} / \mathrm{dL}$ within 2 or 4 weeks if the pretreatment value was low $(\leq 1 \mathrm{mg} / \mathrm{dL}){ }^{7}$ To compare effective drainage between the two groups, the drainage failure rate, which was defined as the failure to achieve successful drainage, was also evaluated. Stent patency was defined as the time between stent placement and stent exchange due to dysfunction. Initial EBD was defined as the primary procedure and the overall EBD included all revised EBDs, including the primary procedure. The definitions of procedure-related complications were modified from those of Cotton et al. ${ }^{18}$ and Freeman et al. ${ }^{19}$ Cholangitis was a serious procedure-related complication and defined as an elevation in total bilirubin with fever $\left(\geq 38^{\circ} \mathrm{C}\right)$ without other infectious etiology. The overall complications included bleeding, pancreatitis, and perforation. A bleeding event was defined when endoscopic hemostasis was required or blood transfusion was needed after a procedure. Post-endoscopic retrograde cholangiopancreatography pancreatitis was defined as new or worsened abdominal pain with an elevation in serum amylase and lipase at least 3-fold the upper limit of normal for 24 hours after a procedure. Perforation was defined as frank perforation during the procedure, free air in an abdominal $\mathrm{X}$-ray after the procedure, or a perforation found in an abdominal image (computed tomography, etc.) performed for abdominal pain evaluation.

\section{Statistical analysis}

The Pearson chi-square test and the Student t-test were used to analyze the statistical significances of different categorical and continuous variables, respectively. Logistic regression was performed initially to analyze successful drainage in the groups. Multilevel logistic regression was used to analyze the overall EBD for repeated EBD procedures after the primary procedure. The long-term effects, indicated by cholangitis events or mortality within 6 and 
12 months after the procedure, were evaluated according to stent type. The mortality rate was estimated using the Kaplan-Meier method and compared using the log-rank test. We calculated the hazard ratios (HR) with $95 \%$ confidence intervals (CI) for developing cholangitis and allcause mortality using a proportional hazards regression model. Since participants could have multiple incident cholangitis, we used Andersen and Gill model to analyze incident cholangitis. The Andersen and Gill modelling is that individuals' failure events after the first are incorporated. In addition, the Andersen and Gill models are variance correction models which account for within-subject correlation of failure times unlike a standard Cox model. ${ }^{20}$ Analysis of study outcomes was performed using timefixed (initial stent) and time-varying variables. In a person whose stent type was changed at least once, time-varying variables were considered to contribute to the initial stent person-time prior to the stent change, and to the next stent person-time after the stent change. To control confounding factors, we adjusted for body mass index, bismuth types (I/II, IIIa/IIIb, and IV), time-varying total bilirubin, prothrombin time, and albumin. When we calculated HR of all-cause mortality by type of stents, we further adjusted for clinical stage in the model. A two-sided p-value of less than 0.05 was considered as statistically significant. Statistical analyses were executed using STATA 15.0 software (StataCorp., College Station, TX, USA).

\section{RESULTS}

\section{Baseline characteristics of the participants}

Of the total 102 patients, BMS were inserted into 35 patients, and MPS were inserted into 67 patients for HMBO caused by hilar cholangiocarcinoma. All the study population was in advanced stage of clinical stage over IIIC or metastatic disease. A comparison of the baseline characteristics of the two groups is presented in Table 1. There were no significant differences between the two groups in median age, sex, underlying disease, or previous history of hepatectomy. Bismuth type IV was predominant in both groups ( $57.1 \%$ in BMS vs $56.7 \%$ in MPS; $\mathrm{p}=0.21$ ). Indexes of liver function status, such as total bilirubin, prothrombin time, and albumin levels, were not significantly different between the two groups. Four patients in the BMS group and six patients in the MPS group underwent palliative chemotherapy. Two patients in the MPS group underwent palliative radiotherapy and photodynamic therapy.

\section{Successful drainage, re-intervention rates, and stent patency}

Table 2 shows the successful drainage rate and comparison of the drainage failure rate in the two types of stents. The successful drainage rate at the initial EBD in the BMS and MPS groups was 71.4\% (25/35) and 65.6\% (44/67), respectively, with no significant difference. There was no significant drainage difference in the two groups in overall EBD analysis. Total bilirubin (crude odds ratio [OR], 1.07; $95 \%$ CI, 1.01 to 1.14 ) and prothrombin time (crude OR, $0.07 ; 95 \% \mathrm{CI}, 0.01$ to 0.81 ) were the factors associated with successful drainage (Supplementary Table 1). A total of $32.4 \%(33 / 102)$ of patients failed to achieve successful drainage at initial EBD and about half of the patients (17/33) underwent external drainage (PTBD) and others $(16 / 33)$ were transferred to another center or hopelessly discharged.

After the initial EBD, the stents were revised in 50.5\% (52/102) of the total study population and the re-intervention rate of the BMS and MPS groups was 40\% (14/35) and $56.7 \%(38 / 67)$, respectively $(\mathrm{p}=0.11)$ (Table 3$)$. The median interval of stent patency was 112 days (77.5 to 143.5 days) in the BMS group and 56 days ( 7 to 201 days) in the MPS group and showed no significant difference $(\mathrm{p}=0.63)$. In the BMS group, only $11.4 \%(4 / 35)$ of the patients revised stents more than twice and $85.7 \%(12 / 14)$ additionally inserted metal-type stents at first re-intervention. In the MPS group, $37.3 \%$ (25/67) of the patients changed stents more than twice and $84.2 \%(32 / 38)$ changed to plastictype stents. Forty-three point eight percent (14/32) of MPS group patients who repeatedly changed to plastic stents more than twice had needed three or more stents due to the complex hilar anatomy. PTBD was done when jaundice did not improve after endoscopic drainage and $26.4 \%(27 / 102)$ of the patients underwent PTBD. The conversion rates to external drainage in the BMS and MPS groups were $22.9 \%(8 / 35)$ and $28.4 \%$ (19/67), respectively $(\mathrm{p}=0.55)$.

\section{Overall complications, post-EBD cholangitis, and 6 and 12-month mortality}

Tables 4 and 5 show the procedure-related complications and survival outcomes. The overall procedure-related complication (bleeding, post-endoscopic retrograde cholangiopancreatography, pancreatitis, and perforation) rate was not significantly higher in the MPS group than the BMS group in the initial EBD (OR, 1.49; 95\% CI, 0.52 to 4.23) nor in the overall EBD (OR, 1.70; 95\% CI, 0.71 to 4.04). However, the incidence of cholangitis was significantly higher in the MPS group than in the BMS group in the initial EBD (HR, 2.89; 95\% CI, 1.57 to 5.29) and in the 
Table 1. Characteristics of the Study Participants in the BMS and MPS Groups

\begin{tabular}{|c|c|c|c|}
\hline Characteristics & BMS group (n=35) & MPS group ( $n=67)$ & $\mathrm{p}$-value \\
\hline \multicolumn{4}{|l|}{ Demographic factors } \\
\hline Sex & & & 0.38 \\
\hline Female & $11(31.4)$ & $27(40.3)$ & \\
\hline Male & $24(68.6)$ & $40(59.7)$ & \\
\hline Age at treatment, yr & $70(62-74)$ & 70 (62-77) & 0.44 \\
\hline $\mathrm{BMI}, \mathrm{kg} / \mathrm{m}^{2}$ & $23.6 \pm 3.5$ & $22.0 \pm 3.4$ & 0.028 \\
\hline Smoking & $14(40.0)$ & 24 (35.8) & 0.68 \\
\hline \multicolumn{4}{|l|}{ Clinical factors } \\
\hline \multicolumn{4}{|l|}{ Underlying disease } \\
\hline Hypertension & $14(40.0)$ & 34 (50.8) & 0.30 \\
\hline Diabetes & $5(14.3)$ & $10(14.9)$ & 0.93 \\
\hline CKD & 0 & $1(1.5)$ & $>0.99 *$ \\
\hline Chronic hepatitis & 0 & $3(4.5)$ & $0.55^{*}$ \\
\hline History of biliary disease & $6(17.1)$ & $10(14.9)$ & 0.77 \\
\hline Previous hepatectomy & 0 & $3(4.5)$ & $0.20 *$ \\
\hline Bismuth type & & & 0.21 \\
\hline I & 0 & $3(4.5)$ & \\
\hline ॥ & $3(8.6)$ & $4(6.0)$ & \\
\hline Illa & $11(31.4)$ & $13(19.4)$ & \\
\hline IIIb & $1(2.9)$ & 9 (13.4) & \\
\hline IV & $20(57.1)$ & $38(56.7)$ & \\
\hline Clinical stage & & & 0.86 \\
\hline IIIc & $9(25.7)$ & 20 (29.9) & \\
\hline IVa & $16(45.7)$ & $27(40.3)$ & \\
\hline $\mathrm{IVb}$ & $10(28.6)$ & 20 (29.9) & \\
\hline Pre-procedure total bilirubin, $\mathrm{mg} / \mathrm{dL}$ & $6.6(2.0-14.1)$ & $5.8(2.3-13.8)$ & 0.85 \\
\hline PT INR & $1.1(1.0-1.1)$ & $1.0(1.0-1.1)$ & 0.73 \\
\hline Albumin, g/dL & $3.6(3.4-4.0)$ & $3.7(3.2-4.1)$ & 0.76 \\
\hline ECOG performance status & & & $0.55^{*}$ \\
\hline 1 & $35(100.0)$ & 64 (95.5) & \\
\hline $2+$ & 0 & $3(4.5)$ & \\
\hline \multicolumn{4}{|l|}{ Treatment factors } \\
\hline Emergent procedure & 0 & $5(7.5)$ & $0.16^{*}$ \\
\hline Treatment & & & 0.76 \\
\hline No treatment & $31(88.6)$ & $59(88.1)$ & \\
\hline Chemotherapy & $4(11.4)$ & $6(9.0)$ & \\
\hline Radiotherapy & 0 & $1(1.5)$ & \\
\hline Photodynamic therapy & 0 & $1(1.5)$ & \\
\hline
\end{tabular}

Data are presented as the number (\%), median (interquartile range), or mean \pm SD.

BMS, bilateral metal stents; MPS, multiple plastic stents; BMI, body mass index; CKD, chronic kidney disease; PT, prothrombin time; INR, international normalized ratio; ECOG, Eastern Cooperative Oncology Group.

*Fisher exact test.

overall EBD (HR, 2.08; 95\% CI, 1.21 to 3.58). Except for the type of stents, the other variables were not significantly associated with cholangitis (Supplementary Table 2).

At 6 and 12 months follow-ups, 37 and 66 participants died, respectively (Fig. 1). The fully-adjusted HR for allcause mortality within 6 months comparing the MPS group with the BMS group was significantly high in the initial EBD (HR, 2.63; 95\% CI, 1.13 to 6.10 ) and in the overall EBD (HR, 2.91; 95\% CI, 1.26 to 6.71) (Supplementary Table 3). Regarding mortality within 12 months, patient in the MPS group were more likely to die compared to the BMS group, while the effect size was relatively smaller than mortality within 6 months and therefore no significant statistical difference was found (Table 5).

\section{DISCUSSION}

In our study, we compared the BMS and MPS for EBD in patients with $\mathrm{HMBO}$ caused by hilar cholangiocarcinoma. There was no significant difference in successful drainage rates, procedure-related complications, or external conversion rates in the two groups. However, in the MPS group, the risk of cholangitis and 6-month mortality 
Table 2. Successful Drainage Rates and Comparison of Drainage Failure Rates

\begin{tabular}{|c|c|c|c|c|}
\hline \multirow{2}{*}{ Rate } & \multicolumn{2}{|c|}{ Initial EBD, OR (95\% CI) } & \multicolumn{2}{|c|}{ Overall EBD, OR (95\% CI) } \\
\hline & BMS & MPS & BMS & MPS \\
\hline Successful drainage, \% (No./total No.) & $71.4(25 / 35)$ & $65.6(44 / 67)$ & & \\
\hline \multicolumn{5}{|l|}{ Drainage failure } \\
\hline Crude & Reference & $1.31(0.54-3.18)$ & Reference & $1.03(0.59-1.81)$ \\
\hline Adjusted & Reference & $1.17(0.44-3.14)$ & Reference & $1.08(0.59-1.67)$ \\
\hline
\end{tabular}

Adjusted for body mass index, bismuth type (I/II, IIla/IIIlb and IV), time varying total bilirubin, PT INR, and albumin.

EBD, endoscopic biliary drainage; OR, odds ratio; Cl, confidence interval; BMS, bilateral metal stents; MPS, multiple plastic stents; PT, prothrombin time; INR, international normalized ratio.

Table 3. Detailed Information on Endoscopic Biliary Drainage Revisions

\begin{tabular}{|c|c|c|c|}
\hline Variable & BMS group $(n=35)$ & MPS group (n=67) & $\mathrm{p}$-value \\
\hline Re-intervention rate & $14(40.0)$ & $38(56.7)$ & 0.11 \\
\hline No. of revision ( $n=52$ ) & & & 0.45 \\
\hline 1 & $10(71.4)$ & 13 (34.2) & \\
\hline 2 & $1(7.1)$ & $10(26.3)$ & \\
\hline 3 & $1(7.1)$ & $5(13.2)$ & \\
\hline 4 & $1(7.1)$ & $5(13.2)$ & \\
\hline 5 & $1(7.1)$ & $1(2.6)$ & \\
\hline 6 & 0 & $1(2.6)$ & \\
\hline 7 & 0 & 2 (5.3) & \\
\hline 10 & 0 & $1(2.6)$ & \\
\hline Type of $2 n d$ stents ( $n=52$ ) & & & 0.001 \\
\hline Metal & $12(85.7)$ & $6(15.8)$ & \\
\hline Plastic & $2(14.3)$ & $32(84.2)$ & \\
\hline Type of last stents ( $n=52$ ) & & & 0.001 \\
\hline Metal & $12(85.7)$ & $12(31.6)$ & \\
\hline Plastic & $2(14.3)$ & $26(68.4)$ & \\
\hline Conversion to PTBD & $8(22.9)$ & $19(28.4)$ & 0.55 \\
\hline Median internal stent patency, day & $112(77.5-143.5)$ & $56(7.0-201.0)$ & 0.63 \\
\hline
\end{tabular}

Data are presented as the number (\%) or median (interquartile range).

BMS, bilateral metal stents; MPS, multiple plastic stents; PTBD, percutaneous transhepatic biliary drainage.

Table 4. Overall Complications and Conversion to External Drainage (PTBD) Risk Depending on the Type of Stent

\begin{tabular}{|c|c|c|c|c|}
\hline \multirow{2}{*}{ Complication and conversion to PTBD } & \multicolumn{2}{|c|}{ Initial EBD, OR (95\% CI) } & \multicolumn{2}{|c|}{ Overall EBD, OR (95\% CI) } \\
\hline & BMS & MPS & BMS & MPS \\
\hline \multicolumn{5}{|l|}{ Overall complication } \\
\hline Crude & Reference & $1.36(0.50-3.68)$ & Reference & $1.58(0.67-3.72)$ \\
\hline Adjusted & Reference & $1.49(0.52-4.23)$ & Reference & $1.70(0.71-4.04)$ \\
\hline \multicolumn{5}{|l|}{ Conversion to PTBD } \\
\hline Crude & Reference & $1.34(0.52-3.46)$ & Reference & $1.07(0.47-2.41)$ \\
\hline Adjusted & Reference & $1.38(0.50-3.79)$ & Reference & $1.17(0.51-2.72]$ \\
\hline
\end{tabular}

Adjusted for body mass index, bismuth type (I/II, IIla/IIIb and IV), time varying total bilirubin, PT INR, and albumin.

PTBD, percutaneous transhepatic biliary drainage; EBD, endoscopic biliary drainage; OR, odds ratio; $\mathrm{Cl}$, confidence interval; BMS, bilateral metal stents; MPS, multiple plastic stents; PT, prothrombin time; INR, international normalized ratio.

rate were higher than in the BMS group.

Previous studies mainly compared single metal stents and single plastic stents or the drainage area (unilateral or bilateral) of the same stent type for hilar obstruction. Similar to the distal biliary obstructions, studies have reported that single metal stents were superior to single plastic stents for successful drainage and patency., ${ }^{6,12,13,15,21}$ Regarding the drainage area, previous studies comparing bilateral with unilateral stents reported some controversial results. Although single stenting is technically easier and less expensive, bilateral stenting is more physiological and has superiority in drainage volume compared to unilateral 
Table 5. Incidence of Cholangitis and Mortality within 6 or 12 Months According to the Type of Stent

\begin{tabular}{|c|c|c|c|c|}
\hline \multirow{2}{*}{ Cholangitis and mortality } & \multicolumn{2}{|c|}{ Initial EBD, HR (95\% CI) } & \multicolumn{2}{|c|}{ Overall EBD, HR (95\% CI) } \\
\hline & BMS & MPS & BMS & MPS \\
\hline \multicolumn{5}{|l|}{ Incidence of cholangitis } \\
\hline Crude & Reference & $2.51(1.41-4.46)$ & Reference & $1.93(1.14-3.25)$ \\
\hline Adjusted & Reference & $2.89(1.57-5.29)$ & Reference & $2.08(1.21-3.58)$ \\
\hline \multicolumn{5}{|l|}{ Mortality within 6 months } \\
\hline Crude & Reference & $1.66(0.80-3.44)$ & Reference & $1.89(0.93-3.82)$ \\
\hline Adjusted & Reference & $2.63(1.13-6.10)$ & Reference & $2.91(1.26-6.71)$ \\
\hline \multicolumn{5}{|l|}{ Mortality within 12 months } \\
\hline Crude & Reference & $1.07(0.65-1.74)$ & Reference & $1.42(0.87-2.31)$ \\
\hline Adjusted & Reference & $1.21(0.65-2.28)$ & Reference & $1.71(0.93-3.13)$ \\
\hline
\end{tabular}

Adjusted for body mass index, bismuth type (I/II, IIla/IIIb and IV), time varying total bilirubin, PT INR, albumin and clinical stage.

EBD, endoscopic biliary drainage; HR, hazard ratio; $\mathrm{Cl}$, confidence interval; BMS, bilateral metal stents; MPS, multiple plastic stents; PT, prothrombin time; INR, international normalized ratio.

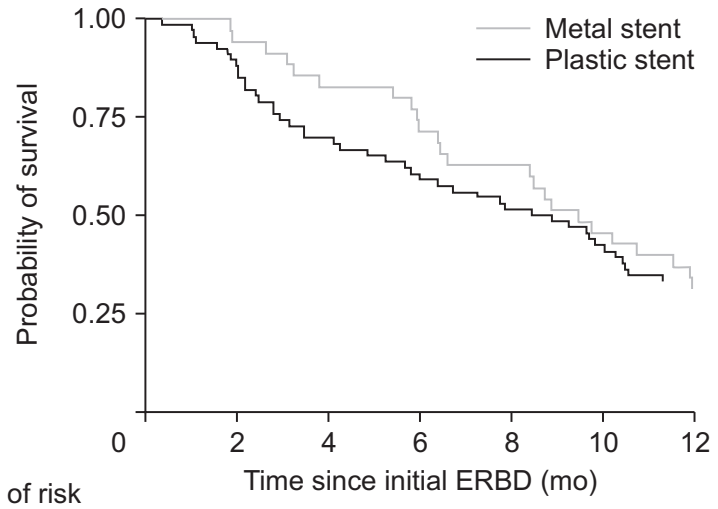

No. of risk Metal stent Plastic stent

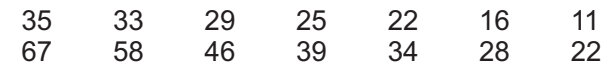

Fig. 1. Survival curves for the bilateral metal stents group and multiple plastic stents group estimated by the Kaplan-Meier analysis. ERBD, endoscopic retrograde biliary drainage.

stenting. ${ }^{22}$ Recent studies have revealed high technical success rates of $90 \%$ to $100 \%$ in bilateral metal stent deployment. ${ }^{14,16,23}$ Naito et al. ${ }^{16}$ reported a retrospective study in 2009 comparing unilateral versus bilateral metal stenting in 46 malignant hilar biliary obstructions. There were no significant differences in successful insertion, drainage and complications between the groups. However, in the cholangiocarcinoma patients, the cumulative stent patency was significantly better in the bilateral stent group $(\mathrm{p}=0.009)$. In 2017, Lee et al. ${ }^{14}$ published a randomized study of 133 malignant hilar biliary stricture patients comparing bilateral versus unilateral metal stents. Unilateral and bilateral drainage by metal stents had similar technical success rates, but bilateral drainage showed more successful drainage ( $95.3 \%$ [61/64] vs $84.9 \%$ [56/66]; $\mathrm{p}=0.047$ ) and resulted in fewer re-interventions and more durable stent patency. However a meta-analysis done by Sawas et al. ${ }^{17}$ concluded that bilateral stenting should be avoided because it has no benefit over unilateral stenting in terms of occlusion rates or therapeutic failures. In summary, the previous studies, recent consensus, and review articles recommended metal stents and considering multi-sectoral drainage for favorable clinical efficacy in high-grade hilar obstruction. ${ }^{10,24}$

To our knowledge, however, there are few studies comparing metal and plastic stent types when two or more stents are needed for HMBO. Although some studies analyzed both stent types and the drainage area, they only compared the same type of stents for the drainage areas. ${ }^{6,15}$ When clinically needed in difficult anatomical variations, such as hilar obstructions, three or more plastic stents can be inserted. However, it is technically difficult to insert more than two BMS. ${ }^{25}$ Regardless of higher re-intervention rates and lower patency, plastic stents also have the advantage that they can be easily removed when the primary obstructing malignant lesions respond to chemotherapy or radiotherapy. Therefore, endoscopic stenting for the malignant hilar obstruction, especially when multiple stenting is needed, is still challenging and the optimal approach has not been identified. In 2020 Xia et al. ${ }^{26}$ reported a most recent study comparing bilateral metal and bilateral plastic stents in HMBO and the BMS group showed better survival, clinical success and longer patency. The study was the first systematic and multicenter study comparing bilateral metal and plastic stents but had limitations of heterogeneities in etiology and difference between the centers. We included homogenous etiology of HMBO and compared overall EBD which included repeated revised procedures. In this study, we compared BMS with bilateral or more than two plastic stents and consistent result was observed that BMS showed better outcomes. We compared the stent types on multiple stentings and the results were consistent with the previous studies reporting that metal stents showed better short-term mortality rates and lower cholangitis risk, both in the initial EBD and the overall EBD than plastic stents, although there was no significant 
difference in achieving successful drainage. Though no significant statistical differences were found in the drainage rate, re-intervention rate, or stent patency between the two groups, the BMS group also showed better trends than those of the MPS groups. Most of the patients in our study underwent EBD before histologic diagnosis. Therefore, the clinicians preferred plastic stents $(67 / 102)$ for the initial EBD when the histologic diagnosis or operability was not confirmed. However, we analyzed the results of the overall EBD by multilevel logistic regression and time-varying variables for patients with repeated endoscopic drainage procedures and the results were similar to the initial EBD results. Therefore, the results apply to primary EBD and also when stent revision is needed.

We should acknowledge that our present study had several limitations. First its retrospective design introduced inherent limitations, including potential selection, measurement, and misclassification biases. In this study, patients who underwent multiple stent revisions were included. Therefore, selection bias was inevitable due to the complexity of individual biliary anatomy and preference of interventionist in each procedure. The long screening duration of study might also lead to chronologic bias. Second, the average follow-up period after endoscopic drainage was short because inoperable stage of patients with poor prognoses were analyzed and the percentage of patients lost to follow-up loss was high because many patients were transferred to other hospice hospitals.

Despite these limitations, this study provides important clinical information. We only included unresectable hilar cholangiocarcinoma patients in a single center with a homogeneous study population and procedures were performed by high volume of experienced endoscopists as strong points. And because we used data from national health insurance system in Korea which all of patients are belonged and death is recorded, the mortality data were reliable. To the best of our knowledge, this is the first study directly comparing MPS with BMS in patients with HMBO caused by cholangiocarcinoma. Although the successful drainage rate was not significantly different between the groups, the BMS group showed lower cholangitis incidence after the procedure and lower 6-month mortality than the MPS group at both the initial EBD and the overall EBD.

In conclusion, for hilar cholangiocarcinoma patients who need palliative EBD procedures for $\mathrm{HMBO}$ with two or more stents, BMS may be recommended over MPS. And when stent revision is needed, BMS may also be preferred over MPS, considering the patient's life expectancy and technical feasibility. However, future studies with data from large sample sizes, longer follow-up periods and prospective designs are warranted to validate our results.

\section{CONFLICTS OF INTEREST}

No potential conflict of interest relevant to this article was reported.

\section{AUTHOR CONTRIBUTIONS}

Study design: J.Y.K, S.L., K.H.L. Data collection: J.Y.K., S.L., J.K.P., K.T.L., J.K.L. Statistical analysis: D.K. Study supervision: K.H.L. Writing of the draft manuscript: J.Y.K., S.L. Critical revision of the manuscript: D.K., J.K.P., K.T.L., J.K.L., D.K.L., K.H.L. All authors approved the final submission.

\section{ORCID}

Jun Young Kim https://orcid.org/0000-0002-9015-9212 Sang-geul Lee https://orcid.org/0000-0003-0471-7511 Danbee Kang https://orcid.org/0000-0003-0244-7714 Dong Kyu Lee https://orcid.org/0000-0002-2353-3905 Joo Kyung Park https://orcid.org/0000-0002-9652-5287 Kyu Taek Lee https://orcid.org/0000-0003-2233-3511 Jong Kyun Lee https://orcid.org/0000-0002-9384-3079 Kwang Hyuck Lee https://orcid.org/0000-0002-5558-0415

\section{REFERENCES}

1. Ballinger AB, McHugh M, Catnach SM, Alstead EM, Clark ML. Symptom relief and quality of life after stenting for malignant bile duct obstruction. Gut 1994;35:467-470.

2. Fukasawa M, Takano S, Shindo H, Takahashi E, Sato T, Enomoto N. Endoscopic biliary stenting for unresectable malignant hilar obstruction. Clin J Gastroenterol 2017;10:485-490.

3. Davids PH, Groen AK, Rauws EA, Tytgat GN, Huibregtse $\mathrm{K}$. Randomised trial of self-expanding metal stents versus polyethylene stents for distal malignant biliary obstruction. Lancet 1992;340:1488-1492.

4. Moss AC, Morris E, Leyden J, MacMathuna P. Do the benefits of metal stents justify the costs? A systematic review and meta-analysis of trials comparing endoscopic stents for malignant biliary obstruction. Eur J Gastroenterol Hepatol 2007;19:1119-1124.

5. Soderlund C, Linder S. Covered metal versus plastic stents for malignant common bile duct stenosis: a prospective, randomized, controlled trial. Gastrointest Endosc 2006;63:986995.

6. Mukai T, Yasuda I, Nakashima M, et al. Metallic stents are more efficacious than plastic stents in unresectable malig- 
nant hilar biliary strictures: a randomized controlled trial. J Hepatobiliary Pancreat Sci 2013;20:214-222.

7. Sangchan A, Kongkasame W, Pugkhem A, Jenwitheesuk K, Mairiang P. Efficacy of metal and plastic stents in unresectable complex hilar cholangiocarcinoma: a randomized controlled trial. Gastrointest Endosc 2012;76:93-99.

8. Kaassis M, Boyer J, Dumas R, et al. Plastic or metal stents for malignant stricture of the common bile duct? Results of a randomized prospective study. Gastrointest Endosc 2003;57:178-182.

9. Levy MJ, Baron TH, Gostout CJ, Petersen BT, Farnell MB. Palliation of malignant extrahepatic biliary obstruction with plastic versus expandable metal stents: an evidence-based approach. Clin Gastroenterol Hepatol 2004;2:273-285.

10. Rerknimitr R, Angsuwatcharakon P, Ratanachu-ek T, et al. Asia-Pacific consensus recommendations for endoscopic and interventional management of hilar cholangiocarcinoma. J Gastroenterol Hepatol 2013;28:593-607.

11. Sangchan A, Chaiyakunapruk N, Supakankunti S, Pugkhem A, Mairiang P. Cost utility analysis of endoscopic biliary stent in unresectable hilar cholangiocarcinoma: decision analytic modeling approach. Hepatogastroenterology 2014;61:11751181.

12. Cheng JL, Bruno MJ, Bergman JJ, Rauws EA, Tytgat GN, Huibregtse K. Endoscopic palliation of patients with biliary obstruction caused by nonresectable hilar cholangiocarcinoma: efficacy of self-expandable metallic Wallstents. Gastrointest Endosc 2002;56:33-39.

13. Raju RP, Jaganmohan SR, Ross WA, et al. Optimum palliation of inoperable hilar cholangiocarcinoma: comparative assessment of the efficacy of plastic and self-expanding metal stents. Dig Dis Sci 2011;56:1557-1564.

14. Lee TH, Kim TH, Moon JH, et al. Bilateral versus unilateral placement of metal stents for inoperable high-grade malignant hilar biliary strictures: a multicenter, prospective, randomized study (with video). Gastrointest Endosc 2017;86:817-827.

15. Liberato MJ, Canena JM. Endoscopic stenting for hilar cholangiocarcinoma: efficacy of unilateral and bilateral placement of plastic and metal stents in a retrospective review of 480 patients. BMC Gastroenterol 2012;12:103.
16. Naitoh I, Ohara H, Nakazawa T, et al. Unilateral versus bilateral endoscopic metal stenting for malignant hilar biliary obstruction. J Gastroenterol Hepatol 2009;24:552-557.

17. Sawas T, Al Halabi S, Parsi MA, Vargo JJ. Self-expandable metal stents versus plastic stents for malignant biliary obstruction: a meta-analysis. Gastrointest Endosc 2015;82:256267.

18. Cotton PB, Lehman G, Vennes J, et al. Endoscopic sphincterotomy complications and their management: an attempt at consensus. Gastrointest Endosc 1991;37:383-393.

19. Freeman ML, Nelson DB, Sherman S, et al. Complications of endoscopic biliary sphincterotomy. N Engl J Med 1996;335:909-918.

20. Guo Z, Gill TM, Allore HG. Modeling repeated time-toevent health conditions with discontinuous risk intervals: an example of a longitudinal study of functional disability among older persons. Methods Inf Med 2008;47:107-116.

21. Wagner HJ, Knyrim K, Vakil N, Klose KJ. Plastic endoprostheses versus metal stents in the palliative treatment of malignant hilar biliary obstruction: a prospective and randomized trial. Endoscopy 1993;25:213-218.

22. De Palma GD, Galloro G, Siciliano S, Iovino P, Catanzano C. Unilateral versus bilateral endoscopic hepatic duct drainage in patients with malignant hilar biliary obstruction: results of a prospective, randomized, and controlled study. Gastrointest Endosc 2001;53:547-553.

23. Iwano $\mathrm{H}$, Ryozawa $\mathrm{S}$, Ishigaki $\mathrm{N}$, et al. Unilateral versus bilateral drainage using self-expandable metallic stent for unresectable hilar biliary obstruction. Dig Endosc 2011;23:4348.

24. Lee TH, Moon JH, Park SH. Biliary stenting for hilar malignant biliary obstruction. Dig Endosc 2020;32:275-286.

25. Yang MJ, Kim JH, Hwang JC, et al. Prospective multicenter study of the challenges inherent in using large cell-type stents for bilateral stent-in-stent placement in patients with inoperable malignant hilar biliary obstruction. Gut Liver 2018;12:722-727.

26. Xia MX, Pan YL, Cai XB, et al. Comparison of endoscopic bilateral metal stent drainage with plastic stents in the palliation of unresectable hilar biliary malignant strictures: large multicenter study. Dig Endosc 2021;33:179-189. 